\title{
Radiologie im Zentrum
}

Die Radiologie hat durch die Entwicklungen des eigenen Fachs wie durch jene der Gesamtmedizin zunehmend eine zentrale Rolle erhalten. Diagnose, Therapie und Therapiekontrolle sowie Nachbeobachtung werden von bildgebenden Verfahren begleitet und beeinflusst. Neue Methoden, wie die Hybridbildgebung, oder Anwendungsgebiete, wie die Elastographie zur Plaqueklassifizierung erweitern das Aufgabengebiet. Allerdings sei eine bessere Zusammenarbeit der jeweils beteiligten Fächer wünschenswert, betonten die Experten anlässlich des European Congress of Radiology (ECR) Anfang März in Wien.

Staging von Krebserkrankungen, Therapieplanung, frühzeitige Therapiebeurteilung und Nachbeobachtung sind wichtige Einsatzgebiete von Hybridbildgebung, die sich aus PET/CT oder PET/MRT zusammensetzt. Die Methoden bieten eine Kombination aus molekularer, biochemischer und struktureller Information, die in dieser Konstellation mehr Aussagekraft erhalten als die Ergebnisse der Einzelmethoden. Damit ist es möglich, die Behandlung von Krebspatienten zu optimieren. Die bei PET-Verfahren in sehr niedriger Dosierung eingesetzten radioaktiven Tracer spielen eine wichtige Rolle, ,die Auswahl der korrekten Tracer und optimierte Protokolle sind von äußerster Wichtigkeit", so Prof. Katrine Riklund, Präsidentin des ECR 2016 und Radiologin und Nuklearmediziner aus Umea/ Schweden. Der Patient profitiert von der Hybridbildgebung durch die Reduktion auf einen einzigen Untersuchungsvorgang, der jedoch eine große Menge an wesentlichen Informationen liefert. "Die Hybridbildgebung hat in der Therapie viel geändert", konstatierte Riklund.

\section{Präzisionsmedizin}

\section{zur Risikostratifzierung}

Der Trend zur personalisierten Medizin, die auf das Individuum zugeschnitten ist, wird zunehmend von der „Präzisionsmedizin" begleitet. Darunter verstehe man die Zusammenfassung von Patienten in Subpopulationen, die von bestimmten Therapien profitieren, erklärte Prof. Gabriel P. Krestin vom Erasmus Medizinzentrum der Universität Rotterdam. „Es geht also um eine Stratifizierung und den Beitrag der Bildgebung zur Risikostratifzierung." Bisher sei die Radiologie sehr beschreibend und subjektiv gewesen, benötigt werden präzise, quantitative Aussagen und Empfehlungen zur Therapie. Hier wird der klinische Nutzen deutlich vergrößert. Die Präzisionsmedizin führe zu einem Paradigmenwechsel.

Präzisionsmedizin sollte daher verschiedene Biomarker basierend auf klinischen, verhaltensbezogenen, genomischen, elektrophysiologischen und bildgebenden Merkmalen integrieren. Die große Bandbreite an Methoden der medizinischen Bildgebung liefert wichtige biologische Informationen über Physiologie, Organfunktionen, Biochemie, Metabolismus, Molekularbiologie und funktionale Genomik. Dabei wird die Möglichkeit, biologische Prozesse zu messen und zu quantifizieren, eröffnet und in der Relation zum anatomischen Bild eine neue Klasse an vielseitigen und nützlichen Biomarkern zur Verfügung zu stellen.„Das Hinzufügen von Genomikdaten erlaubt neue Korrelationen zwischen zellulärer Genomik und Bildgebung auf Gewebeebene", so Krestin. Allerdings sei die Zusammenarbeit zwischen den medizinischen Fächern, die Tests anfordern, und jenen, die sie ausführen, mangelhaft. Benötigt werde ein neues Konzept der integrierten Diagnostik, der Konvergenz aus Bildgebung, Pathologie und Labortests mittels fortgeschrittener Informationstechnik, forderte Krestin.

\section{Im Adipositas-Einsatz}

Der Beitrag der Radiologie zur Bewältigung eines der größten aktuellen Ge- sundheitsprobleme, der Adipositas, kann zu einer besseren Beurteilung des Körperfettanteils und der Lokalisation des Fetts und damit zu einer besseren Risikoeinschätzung führen. Adipositas ist mittlerweile weltweit als Krankheit anerkannt. „Jeder der im Gesundheitssystem arbeitet, ist damit konfrontiert," stellte Prof. Nicholas Finer, Konsulent am University College London Hospital, fest: „Jedes Organ ist davon betroffen." Ein ganz praktisches Problem ist die Durchführung von Untersuchungen adipöser Patienten, da die Geräte oft zu klein sind, um eine ausreichende Bildqualität zu erreichen. Mittlerweile stellt die Radiologie auch bereits minimal invasive Methoden zum Gewichtsverlust bereit.

\section{Ein Ausblick}

Die Elastographie, die bereits in der Unterscheidung von gutartigen von bösartigen Läsionen in unterschiedlichen Organen etabliert ist, wird nun auch für den Einsatz zur Klassifizierung von Plaque untersucht. Dadurch könnte das Risiko von unnötigen Eingriffen verringert werden. Allerdings bestehen derzeit noch technische Einschränkungen und die Reproduzierbarkeit der Ergebnisse ist fraglich. Beispielsweise können kalzifizierte Plaques Probleme bereiten, weil sie einen akustischen Schatten erzeugen und damit schwer zu beurteilen sind.

\section{Wien klin Mag 2016 · 19:55}

DOI 10.1007/s00740-016-0105-6

Online publiziert: 5. April 2016

(c) Springer-Verlag Wien 2016
Quelle: PKERC 2016, 2. März 2016 